\title{
Examining the Effects of a Virtual Character on Learning and Engagement in Serious Games
}

\author{
Vihanga Gamage \\ Technological University Dublin \\ Cathy Ennis \\ Technological University Dublin, cathy.ennis@tudublin.ie
}

Follow this and additional works at: https://arrow.tudublin.ie/scschcomart

Part of the Computer Engineering Commons

\section{Recommended Citation}

Gamage V. \& Ennis C. (2018) Examining the effects of a virtual character on learning and engagement in serious games. Interaction and Games (MIG '18), November 8-10, 2018, Limassol, Cyprus. doi:10.1145/ 3274247.3274499

This Article is brought to you for free and open access by the School of Computer Sciences at ARROW@TU Dublin. It has been accepted for inclusion in Articles by an authorized administrator of ARROW@TU Dublin. For more information, please contact arrow.admin@tudublin.ie, aisling.coyne@tudublin.ie,gerard.connolly@tudublin.ie.

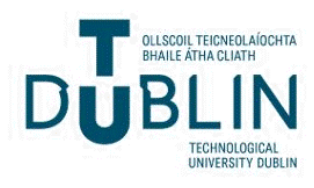




\section{Examining the effects of a virtual character on learning and engagement in serious games}

\author{
Vihanga Gamage \\ School of Computing \\ Dublin Institute of Technology \\ Dublin, Ireland \\ vihanga.gamage@dit.ie
}

\author{
Cathy Ennis \\ School of Computing \\ Dublin Institute of Technology \\ Dublin, Ireland \\ cathy.ennis@dit.ie
}
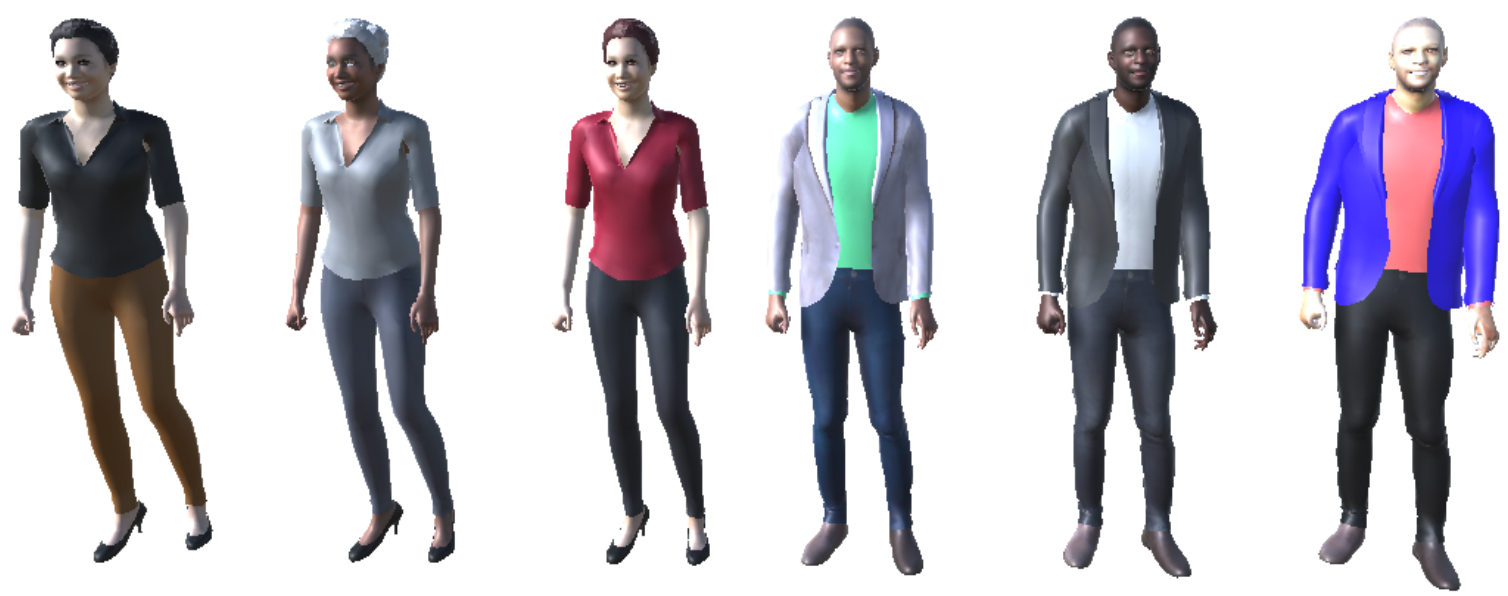

Figure 1: Examples of a number of different character customization combinations.

\begin{abstract}
Virtual characters have been employed for many purposes including interacting with players of serious games, with a purpose to increase engagement. These characters are often embodied conversational agents playing diverse roles, such as demonstrators, guides, teachers or interviewers. Recently, much research has been conducted into properties that affect the realism and plausibility of virtual characters, but it is less clear whether the inclusion of interactive agents in serious applications can enhance a user's engagement with the application, or indeed increase efficacy. In a first step towards answering these questions, we conducted a study where a Virtual Learning Environment was used to examine the effect of employing a virtual character to deliver a lesson. In order to investigate whether increased familiarity between the player and the character would help achieve learning outcomes, we allowed

Permission to make digital or hard copies of all or part of this work for personal or classroom use is granted without fee provided that copies are not made or distributed for profit or commercial advantage and that copies bear this notice and the full citation on the first page. Copyrights for components of this work owned by others than the author(s) must be honored. Abstracting with credit is permitted. To copy otherwise, or republish, to post on servers or to redistribute to lists, requires prior specific permission and/or a fee. Request permissions from permissions@acm.org.

MIG '18, November 8-10, 2018, Limassol, Cyprus

(ㄷ) 2018 Copyright held by the owner/author(s). Publication rights licensed to ACM. ACM ISBN 978-1-4503-6015-9/18/11...\$15.00

https://doi.org/10.1145/3274247.3274499
\end{abstract}

participants to customize the physical appearance of the character. We used direct and indirect measures to assess engagement and learning; we measured knowledge retention to ascertain learning via a test at the end of the lesson, and also measured participants' perceived engagement with the lesson. Our findings show that a virtual character can be an effective learning aid, causing heightened engagement and retention of knowledge. However, allowing participants to customize character appearance resulted in inhibited engagement, which was contrary to what we expected.

\section{CCS CONCEPTS}

- Computing methodologies $\rightarrow$ Perception; • Applied computing $\rightarrow$ Interactive learning environments;

\section{KEYWORDS}

perception, learning, personalization, virtual character

\section{ACM Reference Format:}

Vihanga Gamage and Cathy Ennis. 2018. Examining the effects of a virtual character on learning and engagement in serious games. In MIG '18: Motion, Interaction and Games (MIG '18), November 8-10, 2018, Limassol, Cyprus. ACM, New York, NY, USA, 9 pages. https://doi.org/10.1145/3274247.3274499 


\section{INTRODUCTION}

Virtual Characters have been used extensively over recent years for entertainment, in video games and movies with ComputerGenerated Imagery (CGI). The use of virtual characters in serious applications has also grown steadily, in magnitude and complexity, especially given the increasing ubiquity of computing.

One such example is ECHOES, a serious game that employs an autonomous virtual agent acting as a social partner for children with autism to practice social communication skills [Bernardini et al. 2014]. Gamito et al. [2017] also created a Virtual Reality-based serious game, where a virtual character is used for selective attention tasks in cognitive training on stroke patients. The Virtual Objective Structured Clinical Examination (VOSCE) experience developed by Johnson et al. [2007] to simulate a real human experience for developing interpersonal skills of medical students. The virtual human experience was found to be just as effective as a real human experience during validation; the authors point out that including both speech and gesture recognition in the interface is likely to have been very helpful in achieving this result.

The platform developed by the TARDIS project makes use of virtual characters for game simulations for social training and coaching to develop skills for job interviews [Anderson et al. 2013]. Virtual characters are also employed in solutions developed for intervention of social exclusion, as part of the VERVE project [Taffou et al. 2015].

Another potential use of virtual characters is as pedagogical agents. A pedagogical agent is a conversational agent deployed for the purposes of aiding teaching and learning [Chin et al. 2010]. Studies have been carried out to ascertain the effect of pedagogical agents on learning in the past. However, the results obtained from these studies have been conflicting, and what effect pedagogical agents have on learning, if any, is not clearly known, as demonstrated by the meta-analytic review carried out by Schroeder et al. [2013].

The inclusion of virtual characters in pedagogical applications can provide social context to learning. Virtual characters have been shown to be able to portray human emotions convincingly [Ennis et al. 2013]. In this paper, we investigate whether the fact that virtual characters have been shown capable of emulating human behavior, and that their attributes can be manipulated to alter their appearance, may be harnessed to increase the engagement of users interacting with such a character employed in a pedagogical application.

While studies have been conducted into pedagogical applications employing virtual characters, their conclusions highlight inconsistency with regard to the effect of agent inclusion. In this study, we explore the effect of a virtual character in a pedagogical application, with a knowledge retention task at the core. We posit that the inclusion of a virtual character with human-like behaviour could aid in engaging the user with the application. We also explore the effect of allowing the user to customize aspects of the physical appearance of the character that they will be interacting with.

First, we will discuss some related work that has already been carried out with regard to the perception of virtual characters, pedagogical agents, and customisation of virtual characters. Then, we present the experimental procedure and methods we followed, including details of the system developed, and what data we collected. Following this, we present the hypotheses we made, and the results we collected. Finally, we discuss the results and what future work may follow from this.

\section{RELATED WORK}

\subsection{Perception of Virtual Characters}

Perception of virtual characters has been researched extensively. Humans are quite capable of recognizing the movement of others, and inferring emotions expressed. Johansson et al. [1973] examined motions of a living organism that was represented by means of bright spots depicting the locomotion of the primary joints. It was found that that a recognizable impression could be evoked by with limited information.

Exaggerating movement is a practice that has been widely adopted by animators who intend to create an illusion of life. The effects of rendering styles and facial motion was examined by Hyde et al. [2013]. They concluded that exaggeration affects cartoon and realistic characters differently. Realistic characters were found to be more likeable relative to cartoon characters. They further found that dampening facial motion makes for more apparently intelligent cartoon characters, and realistic characters can benefit from exaggerated facial motion providing more intense and robust communication of emotion.

Research conducted comparing realistic animated virtual characters to those with abstract or cartoonish appearances has shown that realistic characters are perceived differently to abstract characters because they evoke a sense of familiarity [Ferstl et al. 2016]. Creating believable and appealing virtual characters are affected by factors such as design, and rendering of characters, as well as psychological factors such as familiarity [Dill et al. 2012].

\subsection{Existing Pedagogical Agents and Pedagogy}

Pedagogical agents are computer agents designed and built to support education. There have been a number of pedagogical agents developed and evaluated by researchers. In this section, we discuss key theories presented in previous work.

Lester et al. [1997] presented the persona effect. They found that the presence of an animated agent has a strong positive effect on the student's perception of the learning experience, regardless of how expressive the agent is. In its essence, the theory posed by Lester et al. states that the presence of an agent the user can interact with heightens the learning experience.

The persona-zero effect was presented by Miksatko et al. [2010]. This theory suggests that the presence of an agent does not provide any benefit to the performance, but nor does it provide a distraction.

Moreno et al. [2001] observed the 'Personal agent effect'. They deduced that students were more motivated and more interested and that there was increased learning when a social agent was used for the transfer of knowledge than a text-based environment. They displayed an animated agent and a video of a face delivering the lesson and observed a positive efficacy of pedagogy over text or speech used to present the lesson.

Atkinson [2002] presented the 'Embodied agent effect' which resulted in a similar effect to that of the work done by Moreno et al., 
but unlike the work done by Moreno at al., where the agent only had a face, the agent here was embodied and made a monologue delivery while using non-verbal cues.

The results from an examination of pedagogical agent design by Baylor et al. [2004] highlighted that, given realistic agent design seems to affect knowledge transfer positively, there is a value in pedagogical agents representing humans in a realistic manner. The authors point out that while the agent's appearance is a superficial factor in the interface, it plays an important role in affecting learning and motivation, and other factors such as gender, ethnicity and degree of realism should be given due consideration when designing agents.

Johnson et al. [2015] investigated how the concept of pedagogical agents evolved since the year 2000. While their is evidence that they can be beneficial, this observed benefit is not equal across different learning problems and applications, and learner demographics. For instance, one author of this publication takes exception with findings that suggest agents have shown themselves to be more effective at science education compared to language and social education, given that there have been studies carried out where the results demonstrate the opposite. As such, generalized claims on pedagogical agents should be made with care, due to the evident conflicts in results, given that general findings in pedagogical agent research does not seem to hold in some cases even with an increasing body of knowledge in the area; there is much still unknown, and the full potential of pedagogical agents is yet to be realized.

Research in neuro-scientific pedagogy has shown that positive emotion has an effect on learning. Many educational theorists have posed theories of good teaching practices where they highlight that knowledge is best retained when the transfer is done in an environment where strong positive emotion is associated with, and there is no undue stress or anxiety [Krashen 1982]. Similar findings in cognitive psychology deduce that negative emotions such as stress, boredom, confusion and lack of motivation interfere with learning processes [Christianson 1992]. Clinical evidence suggests that while each of these negative emotions are detrimental individually, when combined, the negative effect is profound.

\subsection{Personalization and Customization}

Hefner et al. [2007] has shown that users, during video games where they are personified via an avatar or character, change their self-concept to identify with the character. Players change their self-perception and identify with the role offered in the game. This sense of identification with the game elements has been pointed out as something that brings enjoyment to a player.

Yee and Bailenson [2007] observed The Proteus Effect stating that users are likely to incorporate traits that are representative of the appearance of their character into their in-game behaviour. If their character was physically imposing, they are likely to mimic behaviour associated with this during the game, such as a high level of confidence.

As part of work carried out to investigate factors that can affect the persuasiveness of a virtual salesperson, Hanus et al. [2015] conducted a study where participants were able to customize the appearance of the avatar before it made the sales pitch. Their findings show that customization has a positive impact to how receptive participants would be to a sales pitch, with regard to liking the product more as well as higher purchase intention.

Carrigan et al. [2016] examined the effect of non-player character appearance in a combat game. The study compared two groups of characters, one more aggressive looking than the other (large, red and armed to small, green and unarmed). Both groups had the same performance attributes and were similarly hard to kill. The second group of characters were perceived to be more aggressive, and users tended to perform better against them, possibly because they tried harder to defeat them, and the individuals who played the less-intimidating characters underestimated them. However, the study reported no effect to the enjoyment of the game. These results were consistent with a study conducted by Sell et al. [2009], where they concluded that humans have developed an evolutionary ability to ascertain the strength and fighting ability of an individual by their appearance.

\section{PROCEDURE AND METHODS}

\subsection{Objectives of our study}

As we have described in the previous section, the current research indicates that virtual characters are capable of communicating emotional expression, and that they can be manipulated to affect human perception of them. In this study, we examine if the inclusion of a virtual character in a serious application for learning can have an effect on the perception of the user. We also explore if any such effect also results in an effect to performance of a user, in terms of knowledge retention.

The work carried out by Carrigan et al. has shown that different appearances in characters can affect user behaviour and performance. The Proteus effect and and other research into avatars and user involvement has shown that ownership of aspects of the game has positive effects on the user experience in terms of enjoyment, and confidence. We wish to determine whether allowing users ownership over aspects of the game, and whether being allowed to interact with a character of their own design, has an effect on the perception of the virtual character. Therefore, in this experiment, we also explore if allowing the user to customize aspects of the appearance of the character they interact with, has an effect on perception or performance.

The aim of this experiment was to examine whether delivery of a technical lesson by a virtual character would enhance learning and increase engagement for participants, and whether allowing participants to personalize the physical appearance of the character had any similar effect. We evaluated the effect using participant responses on their perception of the lesson, and by measuring learning via a test. The perceptual effect to engagement was measured by means of questionnaire posed to the user upon completion of the lesson.

\subsection{Experimental Framework}

We used the Unity 3D game development platform to create the stimuli for this experiment. The game had several versions, but lesson material and knowledge retention tested remained constant across each version. The lesson was delivered in a virtual classroom. The virtual character delivered the lesson with the aid of a board 


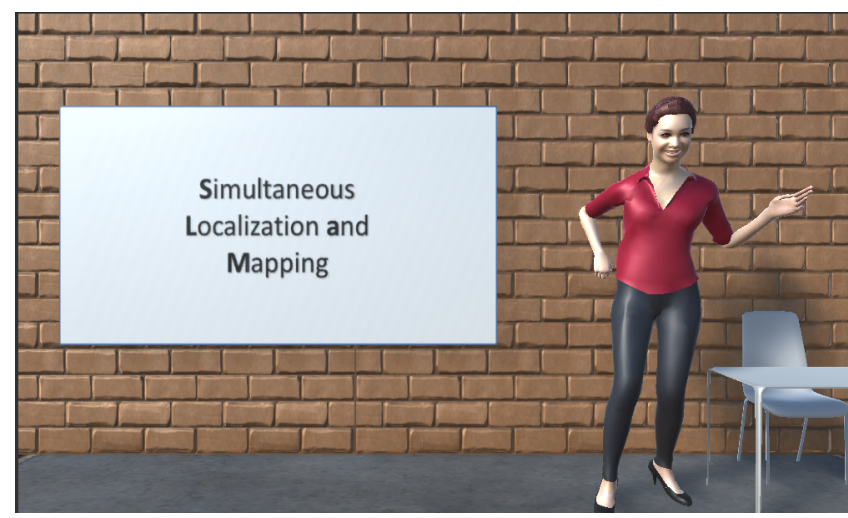

Figure 2: Example classroom scene with a female character.

that displayed diagrams and other supplementary content, as shown in figure 2.

The customization game allowed participants to select between a male and female character, they were then allowed to customize the physical appearance of the character. The participants were presented a live preview of the character reflecting the changes as they customized his/her appearance. The character games included a virtual character, but the participants were assigned a default character. The two customisation screens are as shown in figures 3 and 4.

The voice games consisted of the lesson being delivered without a character present. An overview of the three versions is shown in table 1 . The lesson was narrated by a voice with the same material presented in the boards as the character version, as shown in figure 5.

The lesson was divided into seven parts, as shown in Table 2; four main default parts, and three additional parts that were triggered upon user interaction with the classroom. The hidden levels were expansions to content covered in the main lesson. Material was displayed on the board, and if clicked during the main level, the additional levels were triggered. In the instructions prior to the lesson, participants were encouraged to attempt to interact with the classroom and character by means of clicking. Participants

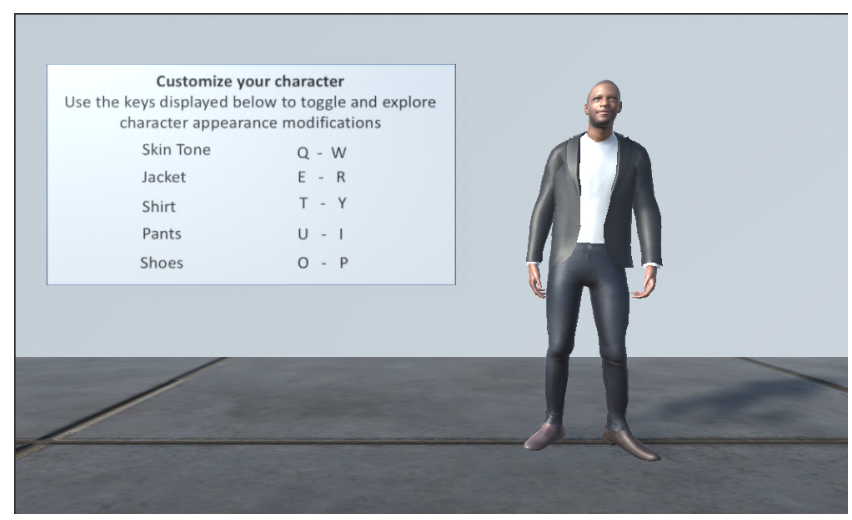

Figure 3: Customization Screen for Male Character.
Table 1: Description of Game Versions.

\begin{tabular}{|l|l|l|}
\hline $\begin{array}{l}\text { Version } \\
\text { Code }\end{array}$ & Version Name & Summary \\
\hline CuG & $\begin{array}{l}\text { Customization } \\
\text { Game }\end{array}$ & $\begin{array}{l}\text { The lesson was delivered by a } \\
\text { virtual character, and the partic- } \\
\text { ipant could customize the char- } \\
\text { acter's appearance. }\end{array}$ \\
\hline ChG & Character Game & $\begin{array}{l}\text { The lesson was delivered by a } \\
\text { virtual character, this character } \\
\text { was either a default male or fe- } \\
\text { male character, assigned at ran- } \\
\text { dom }\end{array}$ \\
\hline VoG & Voice Game & $\begin{array}{l}\text { The lesson was shown on the } \\
\text { same board from the previous } \\
\text { versions, with a randomly as- } \\
\text { signed male voice or female } \\
\text { voice narrating the lesson. This } \\
\text { is the same audio as the voice of } \\
\text { the characters in the character } \\
\text { versions. }\end{array}$ \\
\hline
\end{tabular}

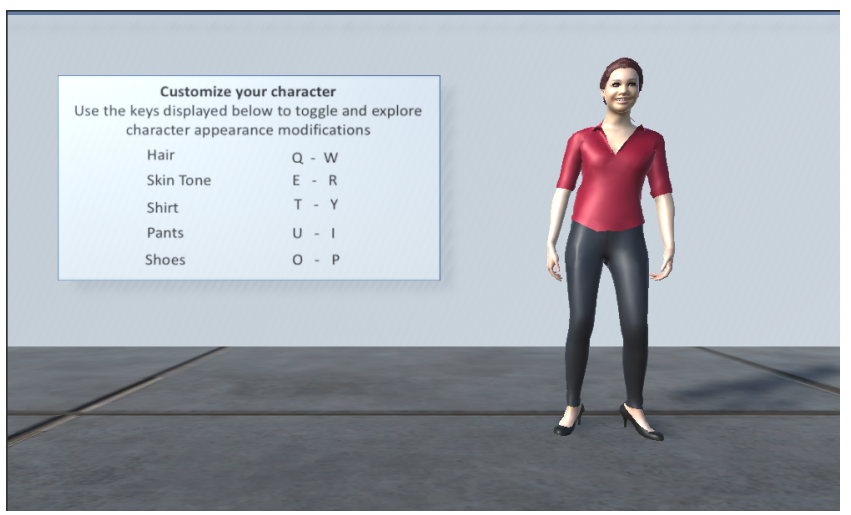

Figure 4: Customization Screen for Female Character.

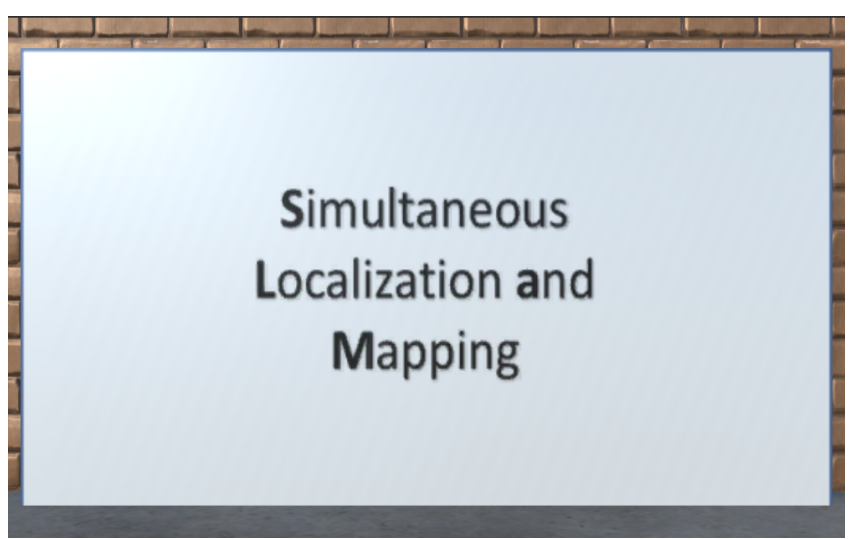

Figure 5: Voice only version with no Character. 
Table 2: Lesson Sections.

\begin{tabular}{|l|l|l|}
\hline Part & Summary & $\begin{array}{l}\text { Number } \\
\text { of Ques- } \\
\text { tions }\end{array}$ \\
\hline Main1 & Introduction to SLAM & 3 \\
\hline Extra1 & Corner Detectors & 1 \\
\hline Main2 & SLAM Approaches & 3 \\
\hline Extra2 & $\begin{array}{l}\text { Proactive vs Lazy } \\
\text { SLAM }\end{array}$ & 1 \\
\hline Main3 & How SLAM works? & 3 \\
\hline Extra3 & $\begin{array}{l}\text { Maximum Likelihood } \\
\text { Estimators }\end{array}$ & 1 \\
\hline Main4 & EKF SLAM & 3 \\
\hline
\end{tabular}

could navigate freely throughout the lesson until they exited to begin the test.

\subsection{Experiment Design and Procedure}

The game was deployed as an online web application. Participants were anonymous, and the consent was given before entry to the game. As a reward for participation, they were included in a raffle for several Amazon vouchers. Participants were recruited through emails and social media and were naïve to the purposes of the study.

Given that the experiment was deployed online, we took measures to ensure data validity. Data collected from participants were validated using metrics on time spent during sections of the game, and samples where the time spent was significantly less than average were excluded. Samples where the answers to test questions were repetitive were also excluded.

$46(36 \mathrm{M}, 10 \mathrm{~F})$ total participant submissions qualified and were used for analysis. 16 participants were in the Customization Game group, 15 in that with the Character Game group, and the remaining 15 in the Voice Game group. None of the participants had extensive knowledge of computer graphics or virtual characters, and did not go beyond playing video games and watching animated movies.

Participants received instructions before commencing the experiment; they were made aware of the ability to navigate freely throughout the lesson, and encouraged to attempt to interact with the character and the classroom.

\subsection{Data Collection}

A questionnaire was used to assess the perceptual effects. They were comprised of a subset of questions from two popular questionnaires; the Game Engagement Questionnaire and the Motivation inventory [Brockmyer et al. 2009; McAuley et al. 1989]. We used a selection

Table 3: Key Questionnaire Items.

\begin{tabular}{|l|l}
1 & I was bored during the lesson. \\
2 & I was fully engaged with the lesson. \\
3 & I felt engaged with the virtual character. \\
4 & $\begin{array}{l}\text { I would prefer this format of informa- } \\
\text { tion delivery to a text-based presenta- } \\
\text { tion. }\end{array}$
\end{tabular}

of items from both of these questionnaires along with questions of our own design. Some of the key questions are shown in table $3 .^{1}$

Assessing the effect on knowledge retention was via a test posed measuring knowledge retained from the lesson. There were questions on the quiz on the content from both the main levels and also the additional hidden levels. We posited that if the participants were highly engaged with the game, they were likely to interact with the game environment more, enter the additional levels and retain knowledge from these sections. A breakdown of the questions from the different levels are displayed in Table 2. The full quiz had 15 questions, 12 of these were on material from the main parts of the lessons, while 3 of these were on material from the hidden parts of the lesson.

\section{HYPOTHESES}

The main impact we wished to measure was that of the inclusion of a virtual character in a learning application. We expected that inclusion of a realistic virtual human would provide a social context to the learning, and that a participant would be be more engaged with a lesson delivered in an application employing a virtual character. Therefore we posited that (H1) Participants were more likely to be engaged to the lesson when the lesson was being delivered through a virtual character, compared to non-character, voice-only control version.

As a result of this heightened engagement, the application employing the virtual character could be more efficient, because increased engagement could lead to enhanced learning. Hence, we hypothesized that (H2) Participants were more likely to perform and retain knowledge better when the lesson was being delivered through a virtual character, compared to non-character, voice-only control version.

We also explored the effect of allowing participants to choose their character, and allowing them to customize aspects of the appearance of that character. We anticipated as a result of increased familiarity and psychological ownership with the character, that participants would be more engaged to the lesson delivered through a personalized character. Therefore, we hypothesized that $(\mathrm{H} 3)$ if allowed to customize the appearance of the virtual character used in a serious application, participants were likely to be more engaged with the character.

\section{RESULTS}

We analysed the data collected to ascertain the effect caused by virtual character inclusion and appearance customisation in serious games. The test scores were analysed to determine the effect to learning, and the participant responses to the questionnaire were analysed to determine the effect to engagement.

We first present the results relevant to the effect on learning, both from character inclusion, and customisation. Then, we present the results on the effect on perception, again, pertaining to both character inclusion, and customisation.

We asked the participants to rate the veracity of the statement "I found the lesson difficult". The average values are displayed in figure 6. They show that each group found the lesson equally hard.

\footnotetext{
${ }^{1}$ The full questionnaire and other supplementary material related to this paper can be found here: www.vihanga.ie/publications/MIG18/
} 


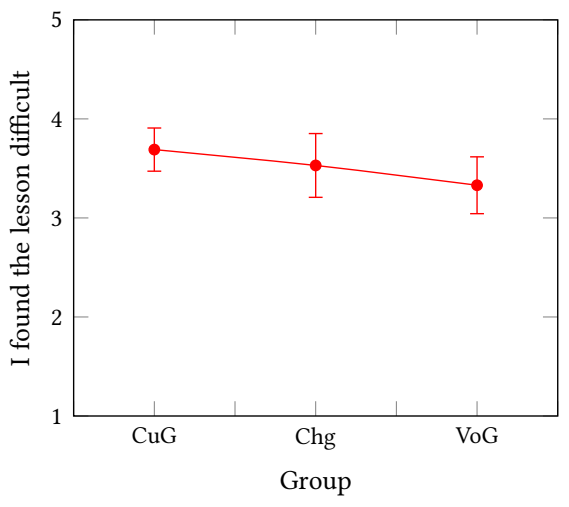

Figure 6: Perceived difficulty of the lesson. The graph shows the mean percentage score and error bars for each point. 1= Strongly Disagree 5= Strongly Agree .

\subsection{Effect on Learning}

We conducted Analysis of Variance (ANOVA) tests on the test scores to determine statistical significance, and post-hoc tests were conducted using the Tukey-Kramer method for multiple comparisons.

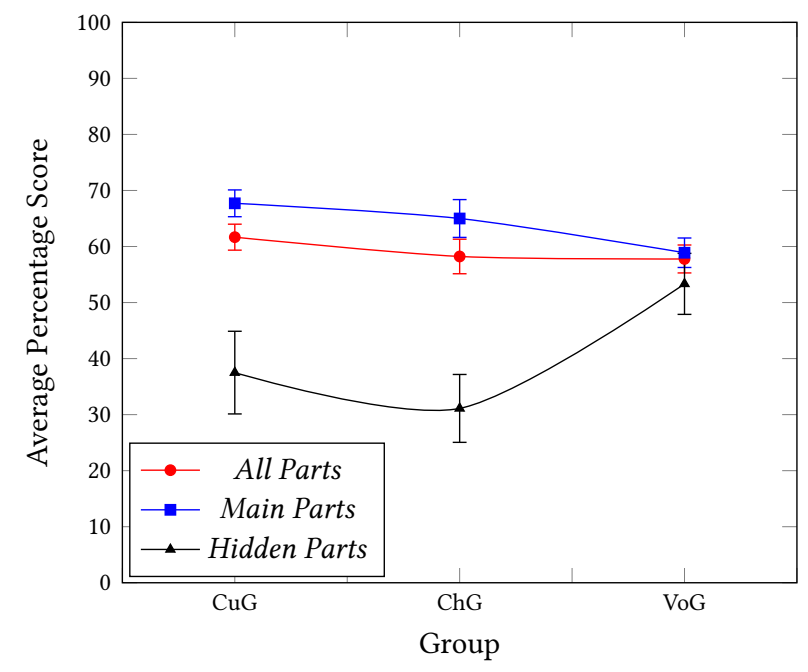

Figure 7: Average Test Scores. The three lines show the respective scores for the full test results, the score of questions on the main part material, and for the questions on the hidden part material.

The three lines shown in figure 10 represent the scores for the three groups for the total 15 minute quiz (All Parts), the 12 questions relating to the material in the main levels (Main Parts), and the 3 questions relating to the material in the extra levels (Hidden Parts).

Character Inclusion. Comparing the $\mathrm{CuG}$ and $\mathrm{ChG}$ group against the VoG group yielded some interesting results. The VoG group were more likely to enter the hidden parts, and their average score for the hidden parts was higher $(F(1,44)=5.835, p<0.05)$ than a combined $\mathrm{CuG}$ and $\mathrm{ChG}$ group. However, the score for the main parts were higher $(F(1,44)=4.758, p<0.05)$ in the combined $\mathrm{CuG}$ and $\mathrm{ChG}$ group than in the VoG group.

Despite the two character groups performing poorly on 'Hidden Parts', the scores for 'All Parts' are relatively close across all three groups, because the higher number of questions in 'Main Parts' gave it a higher weighing in the final score, over the 'Hidden Parts' section of the test.

The normality of the test scores was assessed by using the Shapiro-Wilk test. All the scores were normally distributed, except for the scores of the hidden parts for all three groups, and the score for all parts for the ChG group. In our opinion, the hidden scores were not normally distributed because participants who discover that hidden levels exist have a higher propensity to seek these hidden levels, which leads to higher scores for the hidden parts for these participants, and the opposite holds for participants who were oblivious to the exist of hidden levels. The 'All parts' scores for the ChG group were affected by the skew in the hidden levels for this group; this is likely because more participants in this group were oblivious to the existence of hidden levels, which also lead to the ChG group to have the lowest average score for hidden levels.

Character Appearance Customisation. While the average scores for the $\mathrm{CuG}$ group is marginally higher than the $\mathrm{ChG}$ group, the difference is not statistically significant.

\subsection{Effect on Engagement}

We conducted Kruskal-Wallis $\mathrm{H}$ tests to determine the statistical significance of the questionnaire response data, when comparing three groups. Given that we were testing for multiple hypotheses, we used the Bonferroni correction to compensate for this, therefore reducing the likelihood of incorrectly rejecting the null-hypothesis. The Mann-Whitney U test was used as a post-hoc test for comparing two groups.

Character Inclusion. Three items from the questionnaire were key in ascertaining the effect to engagement. These were perceived boredom, engagement, and preference over a text-based solution. We have presented the average responses for these items in figure 8.

Analysis using the Kruskal-Wallis $\mathrm{H}$ test revealed statistically significant differences in perceived boredom $\left(\chi^{2}(2)=8.702, p<\right.$ $0.0167)$, and perceived preference over a text-based solution $\left(\chi^{2}(2)=\right.$ $9.480, p<0.0167)$. Given that the comparison was across 3 groups, compensation was done through the Bonferroni correction which resulted in a level of significance of $0.0167(p=0.05 / 3)$.

A Kruskal-Wallis $\mathrm{H}$ test of the perceived engagement resulted retention of the null-hypothesis that there is no statistically significant difference when comparison is done across the three sample groups.

When presenting the effect of the character appearance customisation, we ascertained, surprisingly, that participants were much less engaged with the character when it was one whose appearance was customized by the participant. As is demonstrated by the average scores displayed in figure 8, participants perceived the lesson to be less engaging when delivered through a customized character. A post-hoc Mann-Whitney U test between the ChG and VoG 

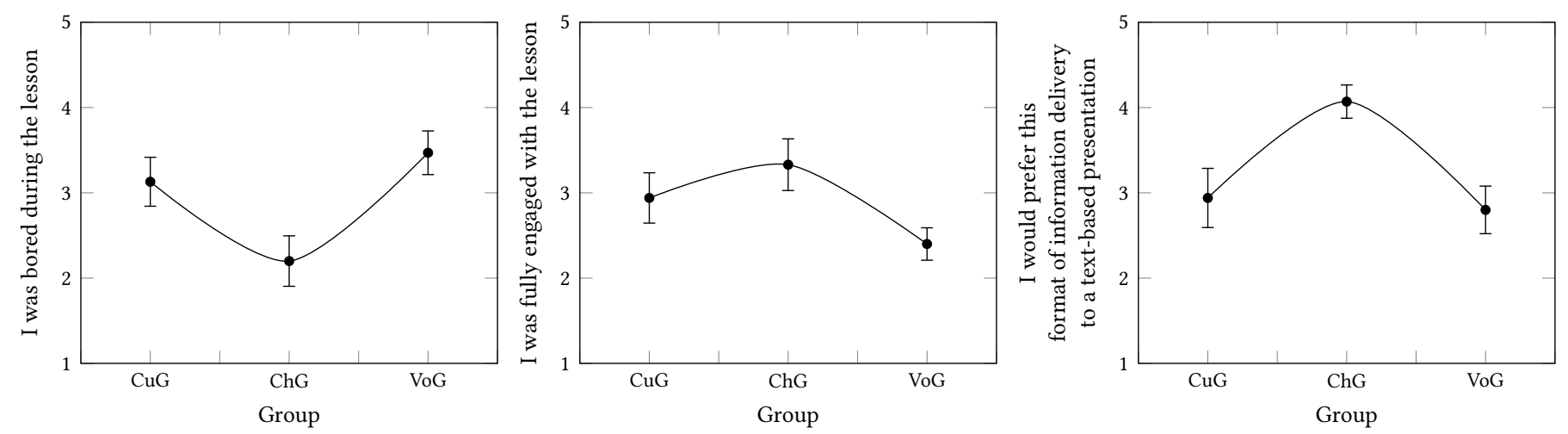

Figure 8: Main items from participant questionnaire: Perceived boredom, engagement, and preference over a text-based solution. 1 = Strongly Disagree, $5=$ Strongly Agree. The graph shows the mean and error bars for each point.

groups to ascertain the effect of character inclusion to perceived engagement with the lesson, resulted in a statistically significant difference $(U=62.5, p<0.05)$.

Character Appearance Customization. We expected allowing a participant to customize the character's appearance would result in increased engagement with the character. This was not the case. The results show that participants were more bored $(U=68.5, p<$ 0.05 ) and less engaged with the lesson when it was delivered by a character customized to their preferences, rather than a default one.

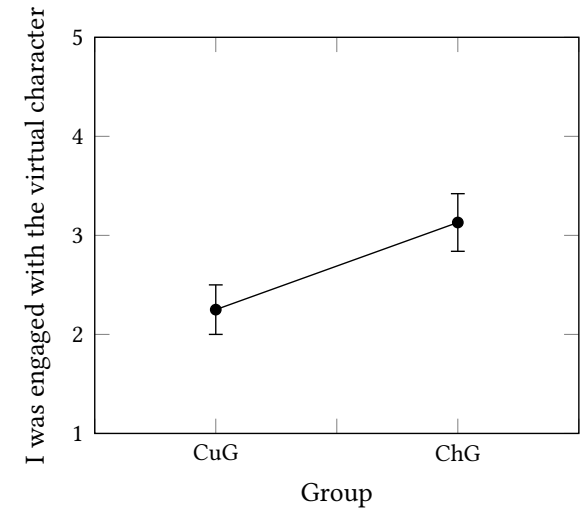

Figure 9: Perceived Engagement with the virtual character. 1 = Strongly Disagree, 5 = Strongly Agree. The graph shows the mean and error bars for each point.

This was further reinforced by the questionnaire item gauging how engaged participants felt with the character itself, as displayed in figure 9 .

There was a statistically significant difference $(U=68, p<0.05)$ that was observed about how engaged participants felt with the character, in the favour of the default character.

Moreover, as can be seen in figure 8 , participants perceived that they would prefer the system with a default character more than one with a customized character, over a text-based solution
$(U=60.5, p<0.05)$. In this regard, the version with a customized character was as unpopular as the voice only version.

\section{CONCLUSION AND FUTURE WORK}

In the study presented in the paper, we attempted to ascertain the effect of character inclusion in a serious game to support learning. We also investigated the effect of allowing participants to customize aspects of the appearance of the character.

Our results show that the inclusion of a character leads to a significant positive effect in the engagement of a participant to the lesson, and that participants are more likely to use a virtual learning environment with a character than one without, in the future, therefore verifying hypothesis $H 1$ as true.

When analysing the test scores to ascertain the effect on learning, participants in the VoG scored higher in the questions related to the extra material trigerred by clicking on the board. On the other hand, there is a statistically significant difference showing higher scores in the main parts for the versions with the character, compared to the VoG group. Based on these observations, we verified hypothesis $H 2$ to be true: participants were more likely to be more engaged with the material when presented through a virtual character, which lead to a positive effect in learning for the main parts. However, the virtual character draws attention from the classroom, which resulted in participants in the character groups to be less likely to notice and click on the board, compared to the VoG.

When examining the effect of allowing customisation of the virtual character, we were not able to verify hypothesis $H 3$ to be true; our results were the opposite to what we had hypothesized. We posited that allowing the participant to select and design the character they interacted with would result in a sense of ownership, and familiarity with the character. We thought that this would translate into heightened engagement in the lesson and character, but the results proved otherwise.

Participants showed a statistically significant decrease in engagement with the character when they were interacting with a customized character, and perceived themselves to be more bored. The results also showed that participants would be much less likely to use a version of the learning environment with the customization relative to the version with a default character. This could 
be because the effort taken to customize the character made the customization seemed like a chore.

In summary, our findings demonstrate that using a virtual character to deliver a lesson results in increased engagement with the material, and that this translates to a positive effect on learning. While allowing customisation of the character does not affect performance, we observed that it inhibited engagement; when including features such as customisation in applications using virtual characters, it should be done in a way that is more integrated and enjoyable, for participants. Also, our findings demonstrate that, when using a virtual character in applications such as those for learning, consideration should be given to the fact that a character is likely to draw attention from the rest of the scene. Therefore, developers might find it more worthwhile focussing on the character, rather than on background elements that might receive little attention from a participant.

In the future, we hope to explore how we can boost ownership and familiarity with a character. A priming process to build a relationship between the character and the participant is an idea we plan to investigate in order to make the customization process more natural, and less laborious. We also plan to investigate factors such as portrayed gender of a character to investigate how user engagement and rapport with a character can be affected by varying character appearance components of pedaogical agent design, as highlighted by Baylor et al. [2004].

In addition, in our future work, we also plan to use participant eye-tracking to investigate where participant attention is focussed on when interacting with a virtual character. Given that our findings in this study showed that the character draws attention from the rest of the scene, further examination of this phenomenon could provide insights on the implications of employing virtual characters, that could be of use when designing applications that deal with them.

The character that was used in this study was fairly basic with regard to realism. The animation was not synchronized to the character speech, and there was no facial animation. In our future work, we hope to build on our findings here by making our character more realistic and examining whether and how we can improve a virtual character to further enhance engagement beyond that of what we observed upon inclusion. Whether increasing engagement would result in a significant increase in performance is of interest. In our future work, we hope to improve the character behaviour to make it adaptive to the participant, and make the character appearance and behaviour more realistic.

\section{REFERENCES}

K. Anderson, E. André, T. Baur, S. Bernardini, M. Chollet, E. Chryssafidou, I. Damian, C. Ennis, A. Egges, P. Gebhard, H. Jones, M. Ochs, C. Pelachaud, K. Porayska-Pomsta P. Rizzo, and N. Sabouret. 2013. The TARDIS framework: Intelligent virtual agents for social coaching in job interviews. Lecture Notes in Computer Science (including subseries Lecture Notes in Artificial Intelligence and Lecture Notes in Bioinformatics) 8253 LNCS (2013), 476-491. https://doi.org/10.1007/978-3-319-03161-3\{_\}35

R. K. Atkinson. 2002. Optimizing learning from examples using animated pedagogical agents. Fournal of Educational Psychology 94, 2 (2002), 416-427. https://doi.org/10. 1037/0022-0663.94.2.416

A. Baylor and Y. Kim. 2004. Pedagogical Agent Design: The Impact of Agent Realism, Gender, Ethnicity, and Instructional Role. (2004), 592-603 pages.

S. Bernardini, K. Porayska-Pomsta, and T. J. Smith. 2014. ECHOES: An intelligent serious game for fostering social communication in children with autism. Information Sciences 264 (4 2014), 41-60. https://doi.org/10.1016/J.INS.2013.10.027
J. H. Brockmyer, C. M. Fox, K. A. Curtiss, E. McBroom, K. M. Burkhart, and J. N. Pidruzny. 2009. The development of the Game Engagement Questionnaire: A measure of engagement in video game-playing. Fournal of Experimental Social Psychology 45, 4 (2009), 624-634. https://doi.org/10.1016/j.jesp.2009.02.016

E. Carrigan, E. Kokkinara, F. Gheorghe, M. Houlier, S. Donikian, and R. McDonnell. 2016. Crowd appearance affects player performance in game combat scenarios. Proceedings of the 9th International Conference on Motion in Games - MIG '16 (2016), 187-192. https://doi.org/10.1145/2994258.2994273

D. B. Chin, I. M. Dohmen, B. H. Cheng, M. A. Oppezzo, C. C. Chase, and D. L. Schwartz. 2010. Preparing students for future learning with Teachable Agents. Educational Technology Research and Development 58, 6 (2010), 649-669. https://doi.org/10.1007/ s11423-010-9154-5

S. Christianson. 1992. Emotional stress and eyewitness memory: a critical review. Psychological bulletin 112, 2 (1992), 284.

V. Dill, L. M. Flach, R. Hocevar, C. Lykawka, S. R. Musse, and M. S. Pinho. 2012. Evaluation of the Uncanny Valley in CG Characters BT - Intelligent Virtual Agents, Yukiko Nakano, Michael Neff, Ana Paiva, and Marilyn Walker (Eds.). Springer Berlin Heidelberg, Berlin, Heidelberg, 511-513.

C. Ennis, L. Hoyet, A. Egges, and R. McDonnell. 2013. Emotion Capture. Proceedings of the Motion on Games - MIG '13 November (2013), 31-38. https://doi.org/10.1145/ 2522628.2522633

Y. Ferstl, E. Kokkinara, and R. McDonnell. 2016. Do I trust you, abstract creature?: a study on personality perception of abstract virtual faces. Proceedings of the ACM Symposium on Applied Perception (2016), 39-43. https://doi.org/10.1145/2931002. 2931014

P. Gamito, J. Oliveira, C. Coelho, D. Morais, P. Lopes, J. Pacheco, R. Brito, F. Soares, N. Santos, and A. F. Barata. 2017. Cognitive training on stroke patients via virtual reality-based serious games. Disability and Rehabilitation 39, 4 (2017), 385-388. https://doi.org/10.3109/09638288.2014.934925

M. D. Hanus and J. Fox. 2015. Persuasive avatars: The effects of customizing a virtual salespersonEEsss appearance on brand liking and purchase intentions. International fournal of Human-Computer Studies 84 (2015), 33-40. https://doi.org/10.1016/j.ijhcs. 2015.07.004

D. Hefner, C. Klimmt, and P. Vorderer. 2007. Identification with the Player Character as Determinant of Video Game Enjoyment BT - Entertainment Computing âĂŞ ICEC 2007, Lizhuang Ma, Matthias Rauterberg, and Ryohei Nakatsu (Eds.). Springer Berlin Heidelberg, Berlin, Heidelberg, 39-48.

J. Hyde, E. J. Carter, S. Kiesler, and J. K. Hodgins. 2013. Perceptual effects of damped and exaggerated facial motion in animated characters. 2013 10th IEEE International Conference and Workshops on Automatic Face and Gesture Recognition, FG 2013 (2013). https://doi.org/10.1109/FG.2013.6553775

G. Johansson. 1973. Visual perception of biological motion and a model for its analysis. Perception \& Psychophysics 14, 2 (1973), 201-211. https://doi.org/10.3758/ BF03212378

K. Johnsen, A. Raij, A. Stevens, D. S. Lind, and B. Lok. 2007. The Validity of a Virtual Human Experience for Interpersonal Skills Education. In Proceedings of the SIGCHI Conference on Human Factors in Computing Systems (CHI '07). ACM, New York, NY, USA, 1049-1058. https://doi.org/10.1145/1240624.1240784

W. Johnson and J. C. Lester. 2015. Face-to-Face Interaction with Pedagogical Agents, Twenty Years Later. International fournal of Artificial Intelligence in Education 26 (2015).

S. D. Krashen. 1982. Principles and Practice in Second Language Acquisition. Vol. 67. 168 pages. https://doi.org/10.2307/328293

J. C. Lester, S. A. Converse, S E. Kahler, S. T. Barlow, B. A. Stone, and R. S. Bhogal. 1997. The Persona Effect: Affective Impact of Animated Pedagogical Agents. Proceedings of the SIGCHI conference on Human Factors in Computing Systems - CHI '97 (1997), 359-366. https://doi.org/10.1145/258549.258797

E. McAuley, T. Duncan, and V. V. Tammen. 1989. Psychometric Properties of the Intrinsic Motivation Inventory in a Competitive Sport Setting: A Confirmatory Factor Analysis. Research Quarterly for Exercise and Sport 60, 1 (3 1989), 48-58. https://doi.org/10.1080/02701367.1989.10607413

J. Miksatko, K. H. Kipp, and M. Kipp. 2010. The persona zero-effect: Evaluating virtual character benefits on a learning task with repeated interactions. Lecture Notes in Computer Science (including subseries Lecture Notes in Artificial Intelligence and Lecture Notes in Bioinformatics) 6356 LNAI (2010), 475-481. https://doi.org/10.1007/ 978-3-642-15892-6\{\}51

R. Moreno, R. E. Mayer, H. A. Spires, and J. C. Lester. 2001. The Case for Social Agency in Computer-Based Teaching : Do Students Learn More Deeply When They Interact With Animated Pedagogical Agents? The Case for Social Agency in Computer-Based Teaching : Do Students Learn More Deeply When They Interact With Anima. Cognition and Instruction 19, 2 (2001), 177-213. https://doi.org/10. 1207/S1532690XCI1902

N. L. Schroeder, O. O. Adesope, and R. B. Gilbert. 2013. How Effective are Pedagogical Agents for Learning? A Meta-Analytic Review. Fournal of Educational Computing Research 49, 1 (2013), 1-39. https://doi.org/10.2190/EC.49.1.a

A. Sell, L. Cosmides, J. Tooby, D. Sznycer, C. von Rueden, and M. Gurven. 2009. Human adaptations for the visual assessment of strength and fighting ability from the body 
and face. Proceedings of the Royal Society B: Biological Sciences 276, 1656 (2009), 575-584. https://doi.org/10.1098/rspb.2008.1177

M. Taffou, J. Ondřej, C. O'Sullivan, O. Warusfel, S. Dubal, and I. Viaud-Delmon. 2015 Auditory-visual virtual environment for the treatment of fear of crowds. Proceedings of the 2015 Virtual Reality International Conference on ZZZ - VRIC '15 (2015), 1-3. https://doi.org/10.1145/2806173.2806176

N. Yee and J. Bailenson. 2007. The proteus effect: The effect of transformed selfrepresentation on behavior. Human Communication Research 33, 3 (2007), 271-290. https://doi.org/10.1111/j.1468-2958.2007.00299.x 